\title{
Characterization of a Coplanar Waveguide Open Stub-Based Discontinuity for MMICs and Filter Applications
}

\author{
Rizwan Masood ${ }^{1}$ and Syed A. Mohsin ${ }^{2}$ \\ ${ }^{1}$ National Engineering and Scientific Commission, Islamabad 44000, Pakistan \\ ${ }^{2}$ School of Electrical Engineering, The University of Faisalabad, Faisalabad 38000, Pakistan \\ Correspondence should be addressed to Rizwan Masood, rmasood@uet-cs.org
}

Received 5 April 2012; Revised 25 June 2012; Accepted 11 July 2012

Academic Editor: Charles Bunting

Copyright ( 2012 R. Masood and S. A. Mohsin. This is an open access article distributed under the Creative Commons Attribution License, which permits unrestricted use, distribution, and reproduction in any medium, provided the original work is properly cited.

\begin{abstract}
This paper presents the analysis and design of an open stub-based two-port discontinuity in a coplanar waveguide. The discontinuity has a good bandpass response and the required design characteristics were achieved by using the method of moments (MOM) and the finite integration technique (FIT). The analysis is found to be computationally efficient which makes the discontinuity suitable for use in complex structure applications. The analysis of the discontinuity is also formulated using the quasi-static finite-difference technique. The proposed discontinuity structure finds applications in RFICs and MMICs. Finally, the application of the discontinuity is supported by the simulated design of a broadband bandpass filter on an MMIC with a relative bandwidth of $96 \%$. The filter is found to have a miniature size and a fairly good stop band rejection.
\end{abstract}

\section{Introduction}

A coplanar waveguide (CPW), first described by Wen [1], has a uniplanar construction and because of this property, it remarkably simplifies fabrication using on-wafer techniques. Since its advent, it is often the preferred choice in many applications especially in RF circuit design including those in Monolithic Microwave Integrated Circuits (MMICs). A detailed description can be found in [2-10]. This is owing to the fact that structure dimensions are not dependent on the substrate thickness for CPW and hence can be chosen to be very small. However, the metallization thickness is critical in the case of CPW since, in contrast to microstrip, this thickness is comparable to the overall dimensions of the structure.

A CPW can be on a single substrate or multiple substrates and, moreover, may be with or without a bottom ground plane in addition to the top semi-infinite ground planes. The name conductor-backed CPW (CBCPW) is used when the bottom ground plane is present. The choice of a particular $\mathrm{CPW}$ topology depends on the specific requirements of an application and its design.
CPW parameters have a small dispersion, that is, they do not vary significantly with frequency since the field is mainly concentrated in the slot regions between the metallization. This leads to increased frequencies up to which static analysis can be considered to be valid in contrast to microstrip or stripline analysis. Dispersion, however, depends on the width of the signal strip, which means that dispersion is high for narrow strips comparable with the skin depth [11]. The CPW supports a quasi-TEM mode of operation but there is no low frequency cutoff unlike the transmission line case.

$\mathrm{CPW}$ discontinuities are an integral part of practical CPW circuits [12]. A number of researchers have worked on CPW discontinuities [13-21]. The CPW open-end, shortend, series gap, step change in the width of center conductor, interdigitated capacitance, and the bend are some of such discontinuities. Some of these discontinuities are shown in Figure 1.

The CPW short-end and open-end series stubs are two important structures for filter applications. The CPW shortend series stub was modeled as a series inductor and the open-end series stub was modeled as a series capacitor by Houdart [3]. These can be attributed to their bandstop and 


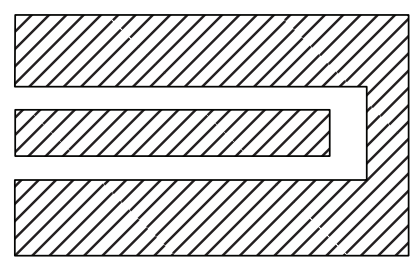

(a)

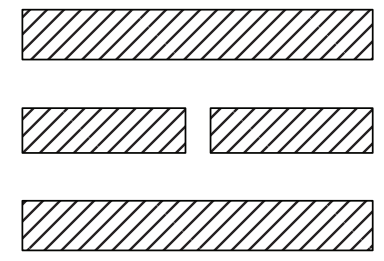

(c)

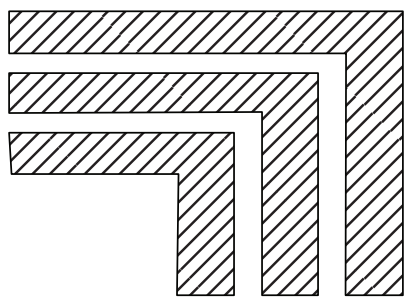

(e)

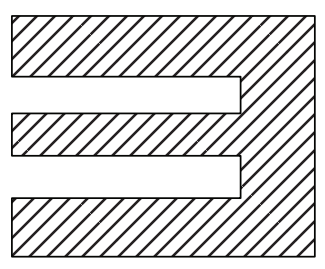

(b)
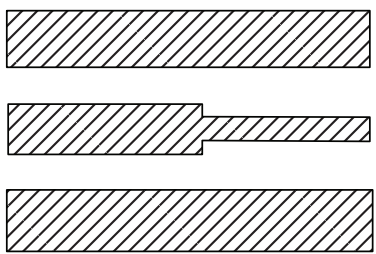

(d)

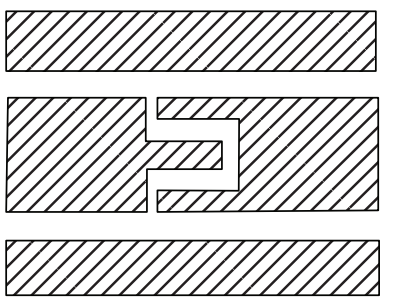

(f)

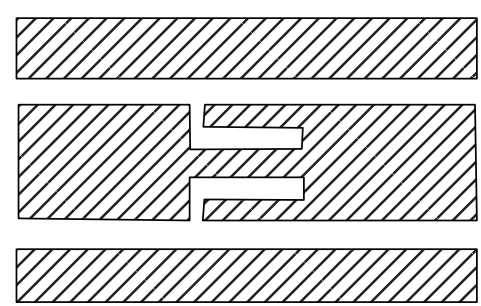

(g)

Figure 1: CPW discontinuities (a), open-end (b), short-end (c), gap (d), step (e), right-angled bend (f), open-end series stub (g) and short-end series stub.

bandpass response, respectively. However, both of these stubs are asymmetric and their resonant nature is valid only for small stub lengths $(l \rightarrow 0)$. Williams and Schwarz [22] extended the model for the open-end stub to a capacitive PImodel and selected a reference plane to remove the element asymmetry.

The open stub-based discontinuity structure presented in this work is symmetric and also has a good bandpass response. This remarkably simplifies the model for use in CAD applications with a significant reduction in computational time and complexity. The fractional bandwidth of the proposed structure is nearly $82 \%$ which is a significant value to utilize the structure in broadband filter applications. This aspect is also demonstrated by a design example at the end of this paper. The CPW single open stub also has a bandpass response but has a very low relative bandwidth compared to a cascade combination of the open stubs as proposed in this work. The resulting cell may be reused or cascaded to build filters with ultrabroadband bandwidths.

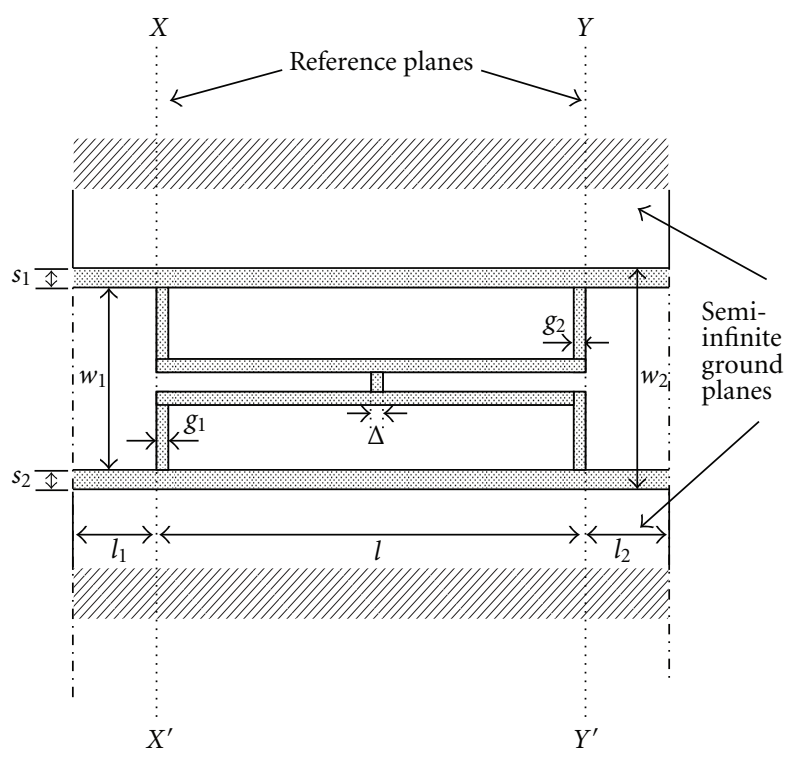

FIGURE 2: The layout of the discontinuity structure unit-cell (DSUC).

The analysis of the proposed discontinuity is also formulated using the quasi-static finite-difference technique. The proposed analysis is also useful in deriving an equivalent network model for the proposed discontinuity. Different authors have discussed the equivalent models for different scenarios concerning CPW [23-27] and may be useful in this regard.

\section{Theory and Simulation}

The layout of the discontinuity structure unit-cell (DS-UC) is shown in Figure 2. The structure is symmetric which provides an advantage as the computational size of the problem is halved for such a structure. $l_{1}$ and $l_{2}$ are the lengths of two uniform coplanar waveguides feeding the DSUC reference planes. Notice the gap dimension $\Delta$ which has a critical influence in achieving the passband response of the DS-UC. $X X^{\prime}$ and $Y Y^{\prime}$ are the reference planes where the field disturbances caused by the discontinuity have decayed down to zero [28]. Hence, $l$ is the effective length of the DS-UC.

The scattering parameters for the discontinuity were obtained by the method of moments, [29], using an electromagnetic (EM) simulator [30]. Only a fundamental even mode has been considered here for simulation (called CPW mode for CPW), whereas the odd mode (called slot mode for CPW) has been suppressed in the simulation. Practically, this is done by using air bridges between the two semiinfinite ground planes and hence keeping them at same potential (i.e., $E$-field is then always oriented from center strip conductor to the semi-infinite ground planes, that is the case for even mode or CPW mode).

The substrate is RT/duroid 6010LM with a dielectric constant of 10.2 and height of $625 \mu \mathrm{m}$ with no bottom ground plane. A magnetic wall was assumed at the input and output ports of the discontinuity where the strip conductor 


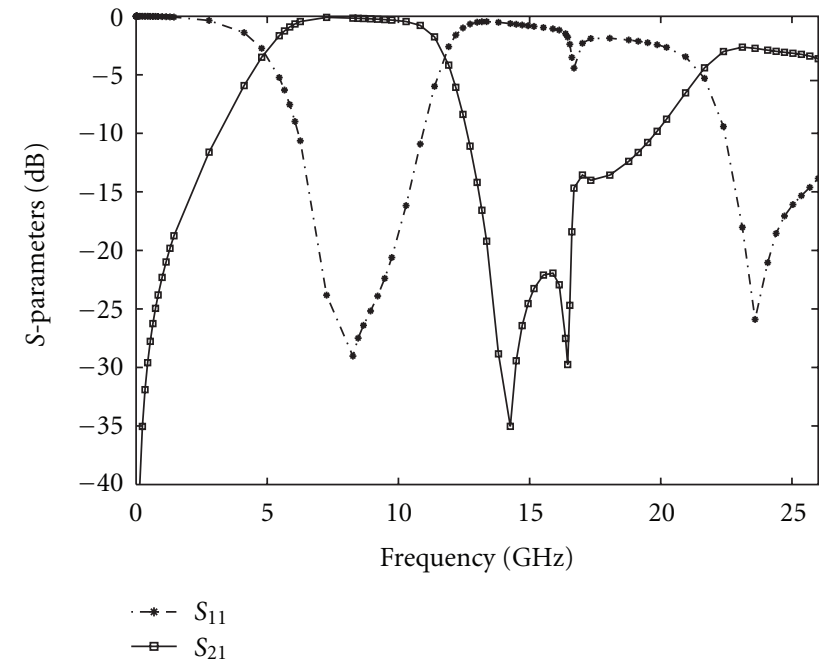

FIGURE 3: Scattering parameters for the DS-UC by MOM. $s_{1}=s_{2}=$ $0.22 \mathrm{~mm} ; g_{1}=g_{2}=0.1 \mathrm{~mm}, \Delta=0.1 \mathrm{~mm}, w_{1}=0.9 \mathrm{~mm}, w_{2}=$ $1.34 \mathrm{~mm} ; l_{1}=l_{2}=0.45 \mathrm{~mm}, l=7.24 \mathrm{~mm}, h=0.635 \mathrm{~mm}, \varepsilon_{r}=$ 10.2 .

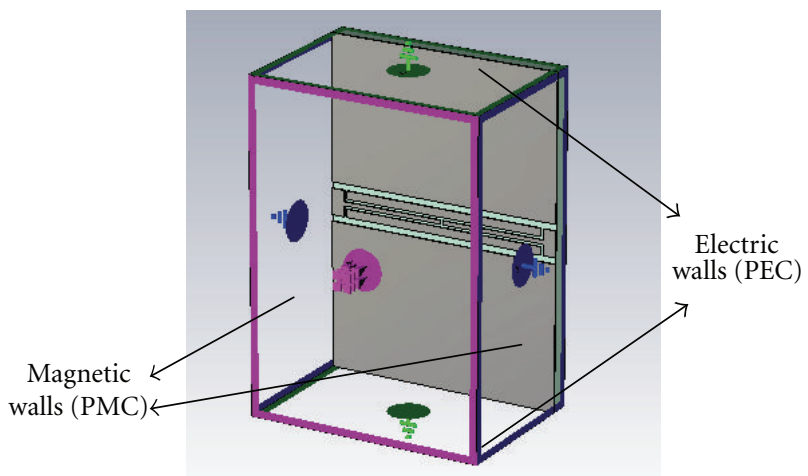

FIGURE 4: Boundary conditions enforced for application of FIT. A perfect magnetic conductor (PMC) was assumed on the side walls where the input and output ports intersect the feeding CPW (marked by blue ground symbols); perfect electrically conducting (PEC) walls were assumed on the top and bottom faces (marked by green ground symbols).

and the shielding would intersect, whereas electric walls were assumed for the remaining boundary conditions. The MOM setup included 257 cells and the computation was completed in a CPU time of 130 seconds only which is an efficient computational overhead for using the proposed discontinuity in complex structure applications. The scattering parameters for the DS-UC are shown in Figure 3. The 3$\mathrm{dB}$ bandwidth of the first passband covers approximately $4.95 \mathrm{GHz}$ to $11.78 \mathrm{GHz}$.

The scattering parameters were verified by the finite integration technique (FIT), using an electromagnetic simulator (CST Microwave Studio) [31]. The boundary conditions used for the numerical setup are shown in Figure 4 and the scattering parameters are shown in Figure 5. The scattering parameters show a good agreement for both the methods

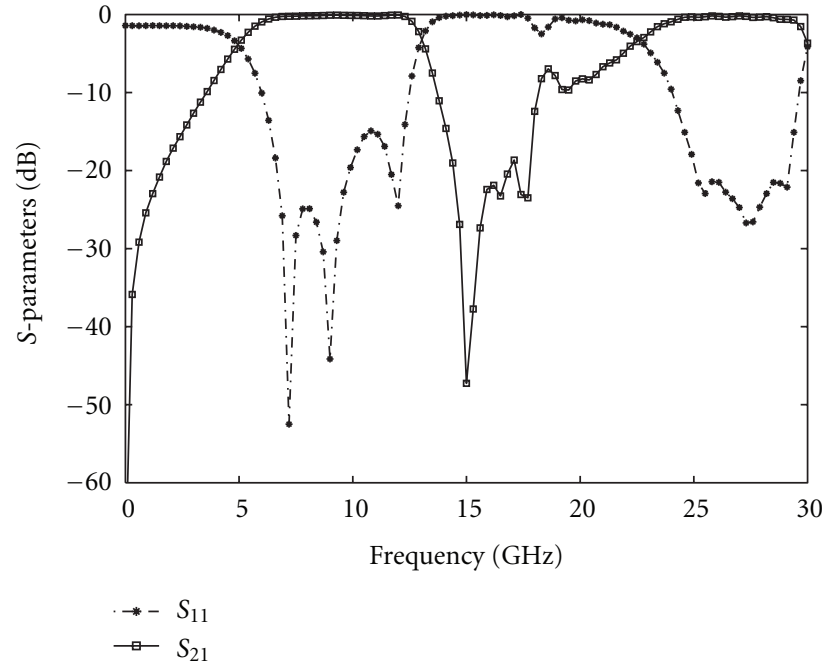

FIGURE 5: Scattering parameters for the DS-UC by FIT. $s_{1}=s_{2}=$ $0.22 \mathrm{~mm} ; g_{1}=g_{2}=0.1 \mathrm{~mm}, \Delta=0.1 \mathrm{~mm}, w_{1}=0.9 \mathrm{~mm}, w_{2}=$ $1.34 \mathrm{~mm} ; l_{1}=l_{2}=0.45 \mathrm{~mm}, l=7.24 \mathrm{~mm}, h=0.635 \mathrm{~mm}, \varepsilon_{r}=$ 10.2 .

which authenticates the validity of the response for the proposed DS-UC.

The effect of the dimensions $g_{1}, g_{2}$, and $\Delta$ on the response of the DS-UC was studied by performing a parameterization sweep and results are shown in Figure 6. The gap dimension $\Delta$ mainly controls the upper cutoff of the passband, whereas the dimensions $s_{1}=s_{2}$ and $g_{1}=g_{2}$ mainly control the fractional bandwidth of passband as is evident from Figures 6(b) and $6(\mathrm{c})$, respectively.

\section{Quasi-Static Finite-Difference Analysis}

The characteristic impedance of a CPW is found through quasi-static analysis by conformal mapping [32-38].

The characteristic impedance and effective dielectric constant for CPW can be computed by quasi-static conformal mapping techniques and given by

$$
Z_{0}=\frac{1}{c C_{a} \sqrt{\varepsilon_{\text {eff }}}}
$$

where $C_{a}$ is the capacitance of CPW with air on both sides (top and bottom) without any intervening dielectric and $\varepsilon_{\text {eff }}$ is the effective dielectric constant.

For the case of CPW on a dielectric substrate of finite thickness, $C_{a}$ and $\varepsilon_{\text {eff }}$ are, respectively, given by

$$
\begin{gathered}
C_{a}=4 \varepsilon_{0} \frac{K\left(k_{1}\right)}{K\left(k_{1}^{\prime}\right)}, \\
\varepsilon_{\mathrm{eff}}=1+\frac{\varepsilon_{r}}{2} .
\end{gathered}
$$

Applying in (1) yields

$$
Z=\frac{30 \pi}{\sqrt{\varepsilon_{\text {eff }}}} \frac{K\left(k_{1}^{\prime}\right)}{K\left(k_{1}\right)},
$$




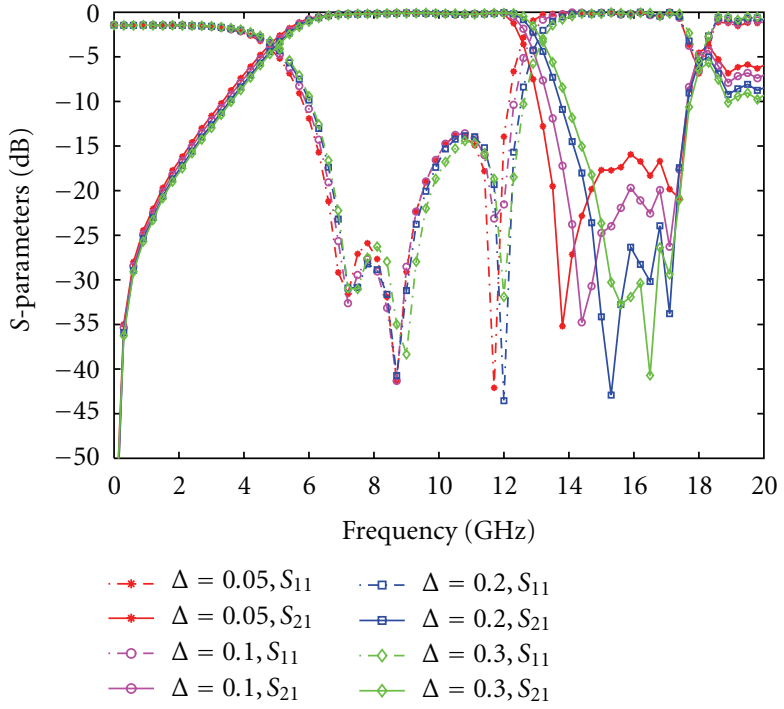

(a)

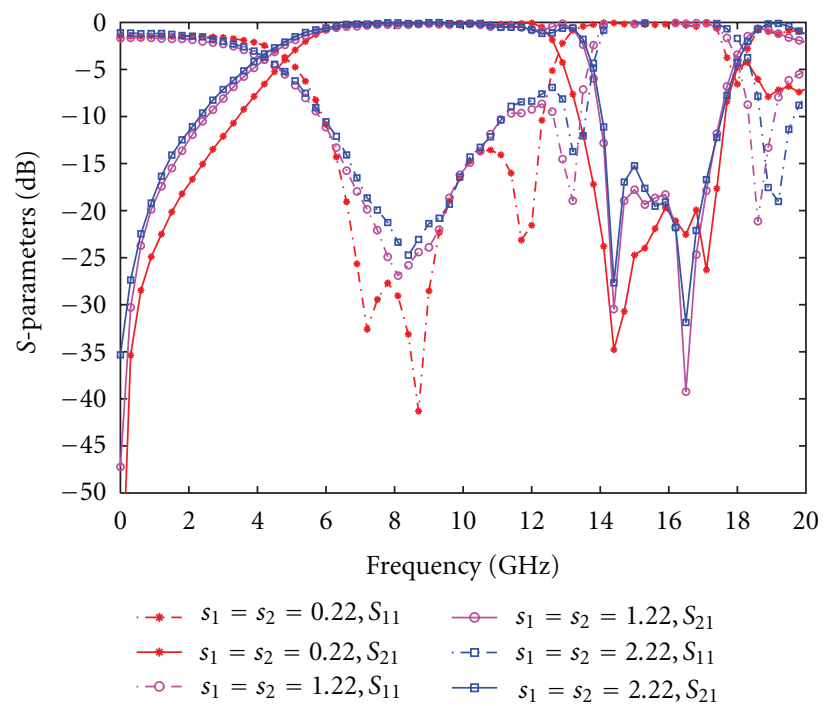

(b)

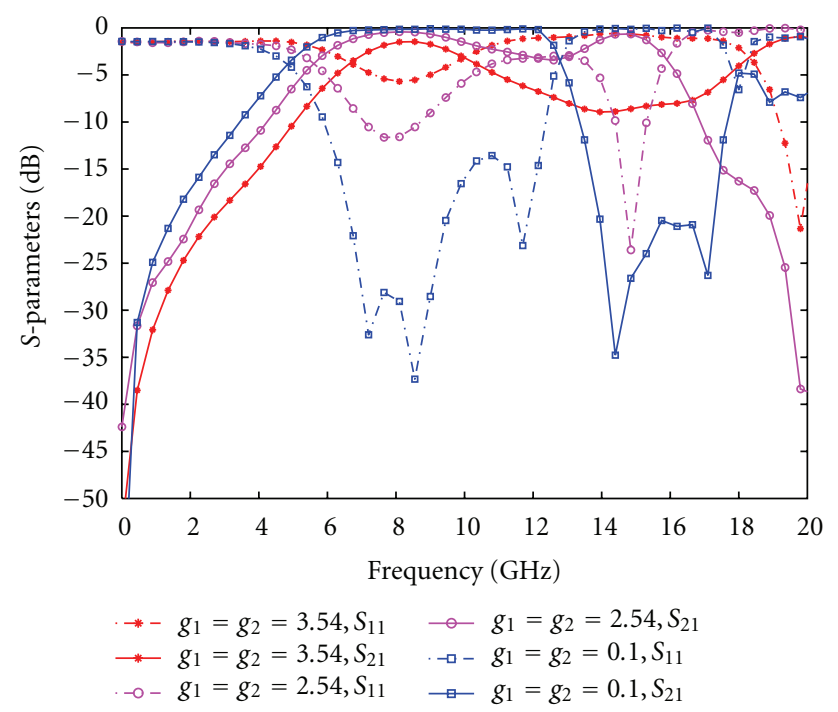

(c)

FIgURe 6: Parameterization of dimensions (a) $\Delta$, (b) $s_{1}, s_{2}$, and (c) $g_{1}, g_{2}$.

where $K\left(k_{1}^{\prime}\right)$ and $K\left(k_{1}\right)$ represent the complete integral of first kind and its complement and $k_{1}$ and $k_{1}^{\prime}$ given by

$$
\begin{aligned}
k_{1} & =\frac{w_{1}}{w_{1}+2 s}, \\
k_{1}^{\prime} & =\sqrt{1-k_{1}^{2}},
\end{aligned}
$$

where $s_{1}=s_{2}=s$.

The ratio between $K^{\prime}(k)$ and $K(k)$ in (3) is an important one in CPW terminology and given by [38] as follows

$$
\begin{aligned}
\frac{K(k)}{K^{\prime}(k)} & =\frac{\pi}{\log _{e}\left[2\left(\left(1+\sqrt{k^{\prime}}\right) /\left(1-\sqrt{k^{\prime}}\right)\right)\right]_{0 \leq k \leq 0.707}}, \\
\frac{K(k)}{K^{\prime}(k)} & =\frac{\log _{e}[2((1+\sqrt{k}) /(1-\sqrt{k}))]}{\pi} \quad 0.707 \leq k \leq 1
\end{aligned}
$$

The characteristic impedance as given by (1) is plotted for the case of $\varepsilon_{r}=10.2$ against the normalized $w_{1} / s$ ratio on a logarithmic scale and is shown in Figure 7. The characteristic impedance of the structure is found to decrease by increasing the ratio $w_{1} / s_{1}$.

The electric field distribution due to the metallization area of the discontinuity gives rise to the capacitance which can be computed by quasi-static finite difference method [28]. The setup for applying the analysis for $E$-field is shown in Figure 8. Magnetic walls were assumed at both the input and output ports for computation of $E$-field (where the strip conductor and shielding intersect). $X X^{\prime}$ and $Y Y^{\prime}$ are the reference planes where the $E$-field caused by the metallization of the discontinuity has decayed to absolute zero and hence they define the geometrical size of the discontinuity. $l_{1}$ and $l_{2}$ 


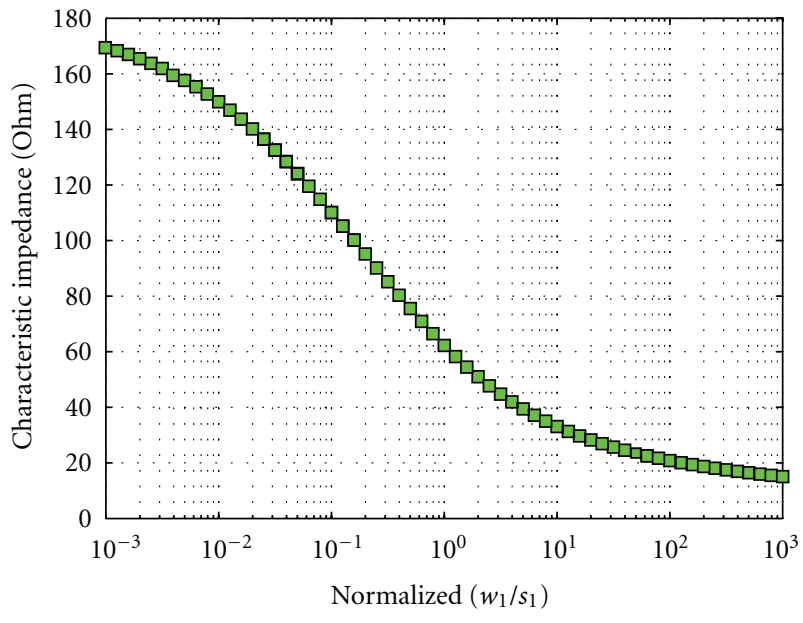

FIGURE 7: Characteristic Impedance of the CPW for the case of $\varepsilon_{r}=$ 10.2 versus normalized $w_{1} / s_{1}$ ratio on logarithmic scale.

are lengths of two uniform coplanar waveguides feeding the discontinuity.

The electric field can be determined from the static electric potential using

$$
\vec{E}=-\vec{\nabla} \varphi,
$$

where $\varphi$ is the static electric potential.

The charges $Q_{1}$ and $Q_{2}$ on the metalized areas with potentials $\varphi_{1}$ and $\varphi_{2}$, respectively, are given by (7) and (8) as follows:

$$
\begin{aligned}
& Q_{1}=\oiint_{A} \vec{D} \cdot \vec{n} d A=\varepsilon_{o} \varepsilon_{r} \oiint_{A} E_{n} d A, \\
& Q_{2}=\oiint_{A} \vec{D} \cdot \vec{n} d A=\varepsilon_{o} \varepsilon_{r} \oiint_{A} E_{n} d A,
\end{aligned}
$$

where $D$ is the electric flux density around the metalized area and $E_{n}$ is the normal component of the electric field. The integration is to be done over the total area $A$ of the metalized region with potentials $\varphi_{1}$ and $\varphi_{2}$, respectively, as shown in Figure 8.

Similarly the charges $Q_{3}$ and $Q_{4}$ deposited on the surface of metalized strips with potentials $\varphi_{3}$ and $\varphi_{4}$, respectively, are given by

$$
\begin{aligned}
Q_{3} & =\oiint_{A} \vec{D} \cdot \vec{n} d A=\varepsilon_{o} \varepsilon_{r} \oiint_{A} E_{n} d A, \\
Q_{4} & =\oiint_{A} \vec{D} \cdot \vec{n} d A=\varepsilon_{o} \varepsilon_{r} \oiint_{A} E_{n} d A,
\end{aligned}
$$

where the integration is to be done over the area as shown in Figure 8 . The area of the strips can be determined since the length $l$ and the gap dimensions $g_{1}$ and $g_{2}$ are known (lengths of sections with potentials $\varphi_{3}$ and $\varphi_{4}$ would be $l-g_{1}-g_{2}$ ).

Similarly, the charges on the semi-infinite ground plane sections with potentials $\varphi_{G}$ and $l_{1}$ and $l_{2}$ (lengths beyond the discontinuity region) are given by

$$
Q_{G s}=\oint_{c} D_{n} d s=\varepsilon_{o} \varepsilon_{r} \oint_{c} E_{n} d s \quad s=1,2,
$$

where the integration is done along a contour in the magnetic walls as shown in the inset of Figure 8.

The net charge $Q_{\text {total }}$ on the discontinuity region (length $l)$ is given by the sum of (7)-(10), that is,

$$
Q_{\text {total }}=Q_{1}+Q_{2}+Q_{3}+Q_{4} .
$$

Finally, the charge stored on the metallization of the discontinuity region is given by the difference of total charge $Q_{\text {total }}$ and the charges on two uniform CPWs, that is, $Q_{G 1}$ and $Q_{G 2}$ as given by (11).

Assuming the potential difference between the CPW structure representing the overall discontinuity region and the ground planes is given by

$$
V=\varphi_{D}-\varphi_{G} .
$$

Then the capacitance of the discontinuity region would be given by

$$
C_{D}=\frac{Q_{D}}{V}=\frac{Q_{\text {total }}-Q_{G 1}-Q_{G 2}}{V} .
$$

The E-field distribution plotted using CST Microwave Studio is shown in Figure 9 with respect to $x, y$, and $z$ axes. Port 1 was excited from the left under a quasi-TEM mode to investigate the electric field distribution inside the slotted region of discontinuity structure. Most important is the component $E_{y}$ since the structure has been placed along $x z$-plane. $E_{y}$ shows a strong electric field distribution near the gap discontinuity which decays down as we move towards the ends of structure from the center.

The magnetic field distribution of the discontinuity gives rise to the inductance so the computation of magnetic field intensity will give the equivalent inductance. The setup for computing the static magnetic field is shown in Figure 10.

The magnetic field can be computed from the static magnetic potential using

$$
\vec{H}=-\vec{\nabla} \psi
$$

The magnetic field distribution inside the discontinuity is computed using the quasi-static finite difference method as given in [28].

The magnetic flux through the discontinuity region is given by

$$
\phi_{T}=\iint_{A} \vec{B} \cdot \vec{n} d A=\mu_{o} \iint_{A} H_{n} d A,
$$

where the integration is to be done along the slot region over the area $A$ as shown in Figure 10. The assumption made was that the metallization thickness is negligible $(t \rightarrow 0)$ and the substrate material has no effect on magnetic field due to the metallization, that is, $\mu_{r}=1$.

Likewise, the magnetic flux due to the two uniform coplanar waveguides of lengths $l_{1}$ and $l_{2}$ is given by

$$
\phi_{G t}=\int_{\mathcal{c}} B d s=\mu_{o} \int_{\mathcal{c}} H d s \quad t=1,2 .
$$

The magnetic flux due to the discontinuity metallization is given by the difference of the total magnetic flux and the 


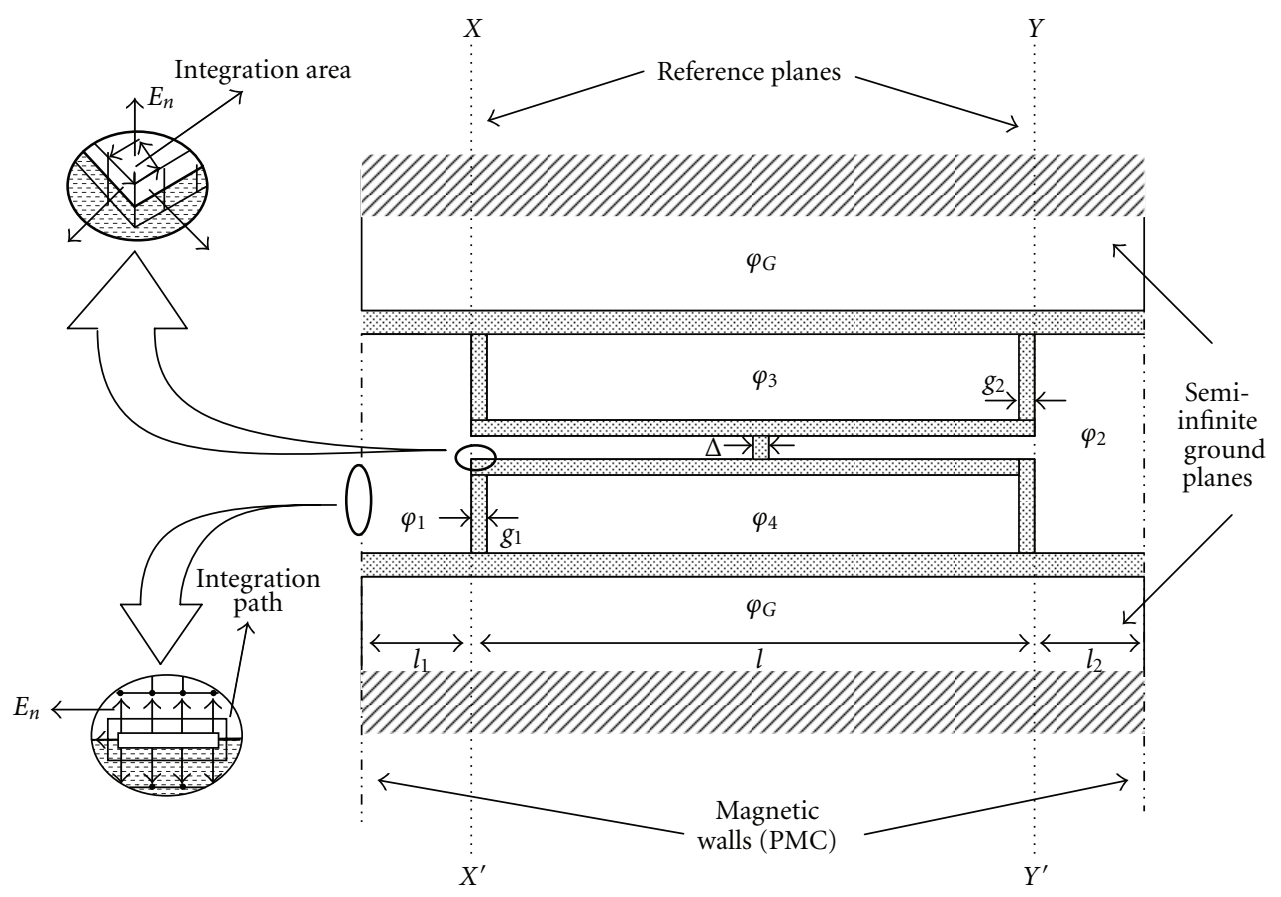

FIGURE 8: Setup for E-Field computation, $l_{1}=l_{2}=0.45 \mathrm{~mm}, g_{1}=g_{2}=0.1 \mathrm{~mm}$, and $l=7.24 \mathrm{~mm}$.

magnetic flux due to the uniform CPWs (lengths $l_{1}$ and $l_{2}$ ), that is,

$$
\phi_{D}=\phi_{T}-\phi_{G 1} l_{1}-\phi_{G 2} l_{2} .
$$

From the net magnetic flux, the current in the centre conducting plane can be determined as follows:

$$
I=\int_{\mathcal{c}} \vec{H} \cdot \vec{d} s=\psi_{T}
$$

where the line integral is to be taken along the electric wall shown in Figure 10.

Now the equivalent inductance due to the discontinuity can be evaluated as follows:

$$
L_{D}=\frac{\phi_{D}}{I} .
$$

The magnetic field distribution inside the substrate under the quasi-TEM mode assumption was plotted using CST Microwave Studio and is shown in Figure 11, which shows that the magnetic field is stronger near the ends of discontinuity in contrast to the electric field distribution, which is concentrated at the centre of the gap discontinuity.

To support the fact that electric field is concentrated at the middle of gap discontinuity and magnetic field near to the ends of discontinuity, the electric and magnetic field energies $\left(\mathrm{J} / \mathrm{m}^{3}\right)$ are observed using CST Microwave Studio and are shown in Figure 12 where the peak electric energy density $=0.0872 \mathrm{~J} / \mathrm{m}^{3}$ and peak magnetic energy density $=$ $0.0459 \mathrm{~J} / \mathrm{m}^{3}$

To support the usefulness of the discontinuity structure for filter applications, a bandpass response with a good rolloff was achieved by cascading three cells of DS-UC as shown in
Figure 13. The structure had a miniature size (a sectional area of $1.34 \times 24 \mathrm{~mm}^{2}$ ).

The scattering parameters for the bandpass structure were verified by both the method of moments, using Momentum [30], and FIT using another simulator, CST Microwave Studio [31]; these are shown in Figures 14 and 15, respectively. The relative bandwidth of the bandpass structure was computed to be $96 \%$. MOM setup for the computation of bandpass filter utilized a processor overhead of $71.42 \mathrm{MB}$ and a CPU time of 22 minutes and 6 seconds for computation of the final results.

The bandwidth of the filter can be controlled by adjusting the dimensions $\Delta, s_{1}, s_{2}, g_{1}$, and $g_{2}$ of the unit cell. This can be done by first designing a unit cell for the desired bandwidth and then cascading multiple cells for desired roll-off. The design of individual cell can be done using perturbation of dimensions $\Delta, s_{1}, s_{2}, g_{1}$, and $g_{2}$. This can be achieved by performing a parametric study of the dimensions $\Delta, s_{1}, s_{2}, g_{1}$ and $g_{2}$ using the simulator program. A rule of thumb as mentioned already is that the gap dimension $\Delta$ mainly controls the upper cutoff of the passband, whereas the dimensions $s_{1}=s_{2}$ and $g_{1}=g_{2}$ mainly control the fractional bandwidth of passband as illustrated in Figure 6 above.

Once a unit cell is designed, a filter with desired bandwidth can be designed by cascading multiple sections for desired rolloff. As in our case, cascade of two cells provided an acceptable rolloff.

\section{Conclusions}

An open stub-based two-port discontinuity in a coplanar waveguide is proposed with a much better return loss and relative bandwidth than the single open stub section. The 
$(\mathrm{V} / \mathrm{m})$

$(\mathrm{A} / \mathrm{m})$

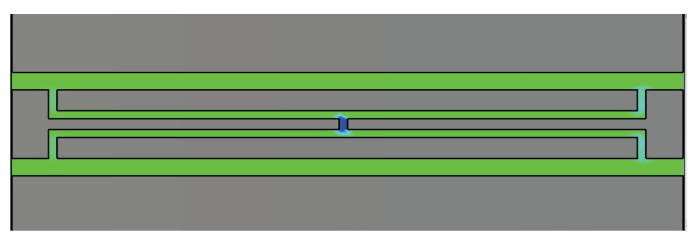

44249
34570
26273
17976
9680
0
-6914
-15211
-23507
-31804
-44249

(a)

$(\mathrm{V} / \mathrm{m})$

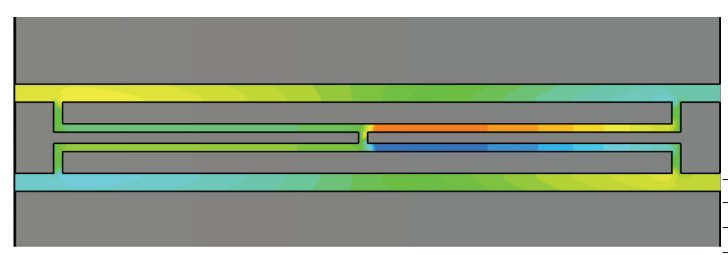

49303
38518
29273
20029
10785
0
-7704
-16948
-26192
-35436
-49303

(b)

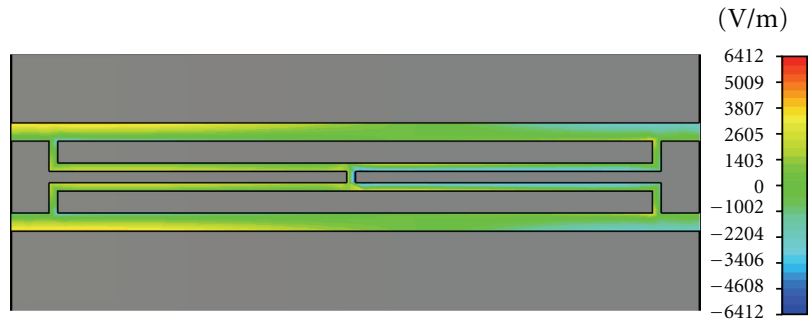

(c)

Figure 9: Electric field $(\mathrm{V} / \mathrm{m})$ distribution inside the discontinuity structure at $8 \mathrm{GHz}$ under quasi-TEM fundamental mode (a) $E_{x}$, (b) $E_{y}$, and (c) $E_{z}$.

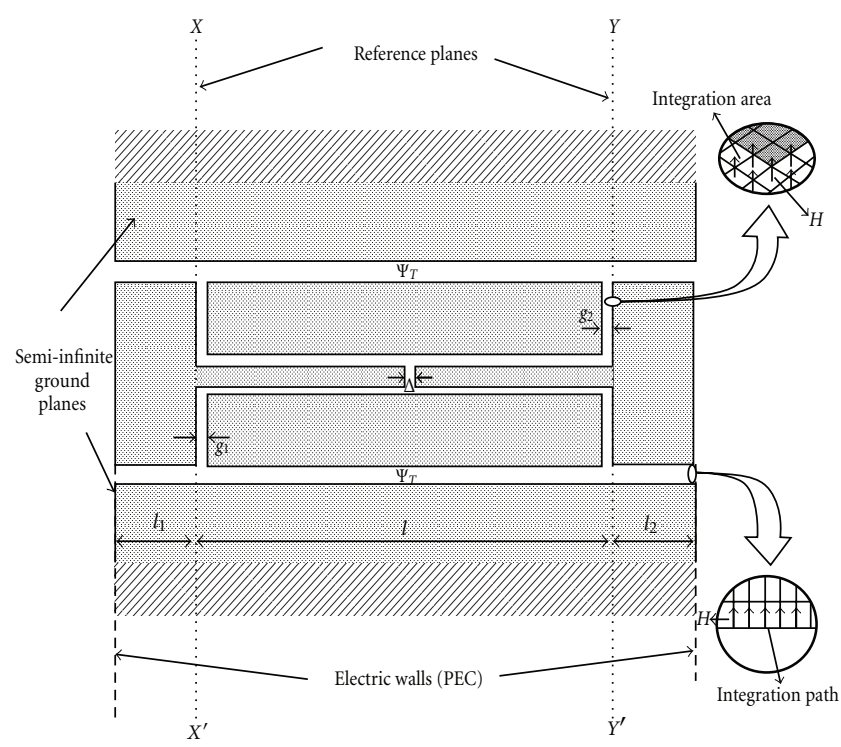

FIgURE 10: Setup for magnetic field computation, $l_{1}=l_{2}=$ $0.45 \mathrm{~mm}, g_{1}=g_{2}=0.1 \mathrm{~mm}$, and $l=7.24 \mathrm{~mm}$.

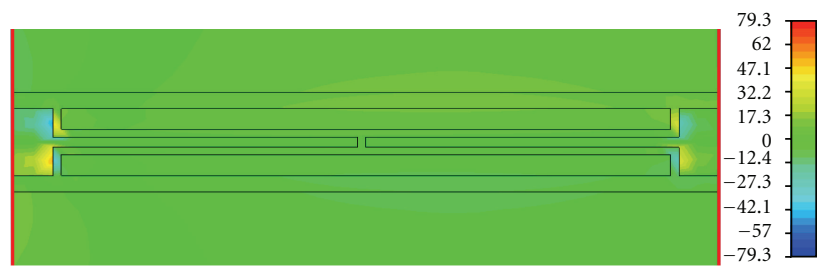

(a)

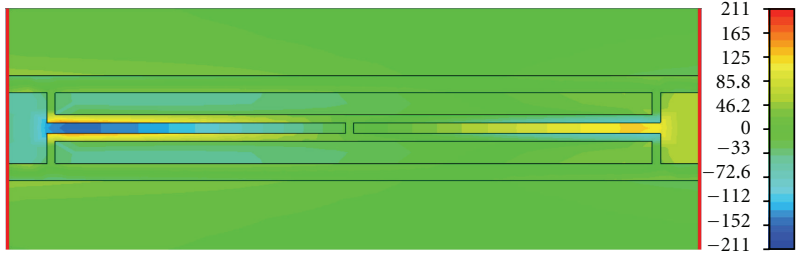

(b)

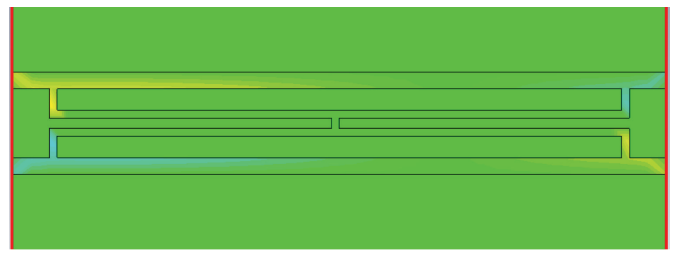

$(\mathrm{A} / \mathrm{m})$

(c)

Figure 11: Magnetic field (A/m) distribution inside the discontinuity structure at $8 \mathrm{GHz}$ under quasi-TEM fundamental mode (a) $H_{x}$, (b) $H_{y}$, and (c) $H_{z}$.

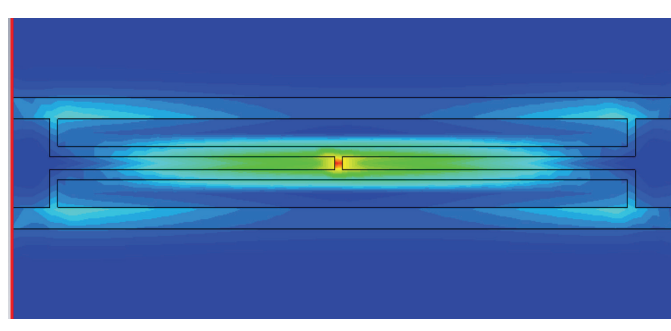

$\left(\mathrm{J} / \mathrm{m}^{3}\right)$ 0.0598 0.0532 0.0476 0.042 0.0364 0.0308 0.0252

0.0196

0.014 0.0084 $2.99 e-009$

(a)

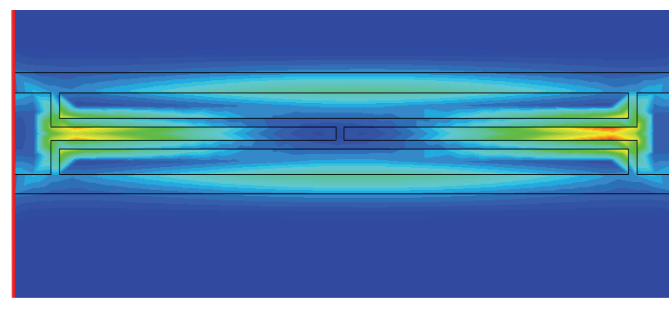

$\left(\mathrm{J} / \mathrm{m}^{3}\right)$

0.032

0.0285

0.0255

0.0225

0.0195

0.0165

0.0135

0.0105

0.00751

0.00451

$.25 e-006$

(b)

Figure 12: Energy density $\left(\mathrm{J} / \mathrm{m}^{3}\right)$ under fundamental mode (a) electric energy density and (b) magnetic energy density. 


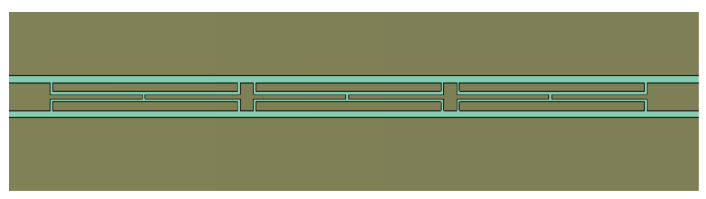

Figure 13: Top view of bandpass structure achieved by cascading three cells of DS-UC.

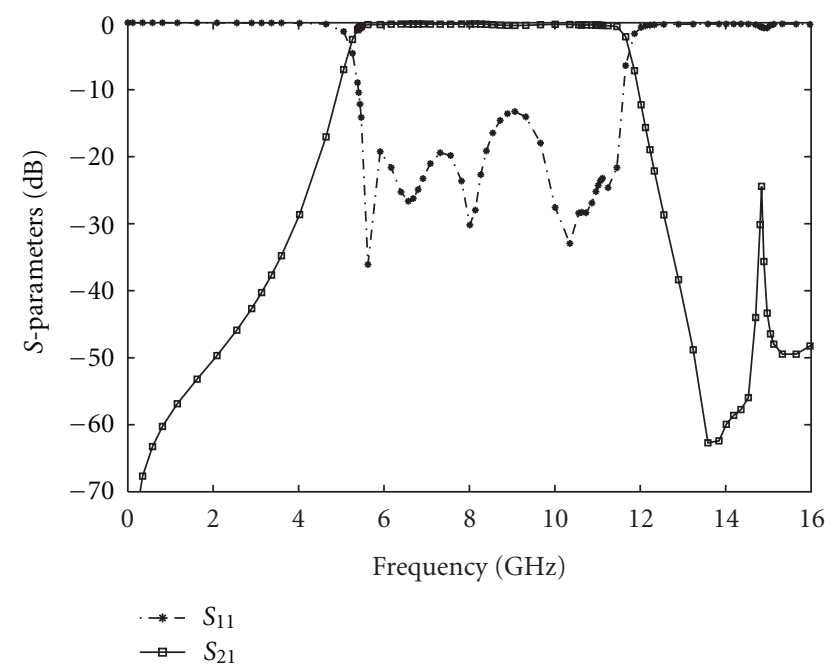

FIGURE 14: Scattering parameters for the bandpass resonator constructed by cascading three cells of DS-UC by MOM.

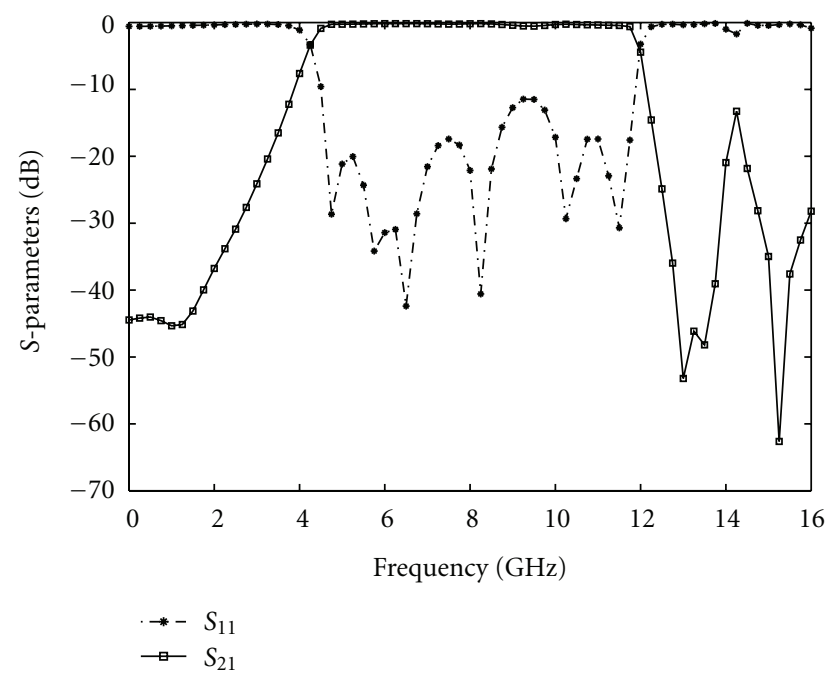

FIGURE 15: Scattering parameters for the bandpass resonator constructed by cascading three cells of DS-UC by FIT.

agreement between the results obtained by the classical methods of computational electromagnetics, that is, MOM and FIT supports the validity of the structure's usefulness. The discontinuity has a good bandpass response and an efficient computational overhead which makes it suitable for embedding in complex structure applications. The analysis of the discontinuity was also formulated using the quasistatic finite-difference technique. The proposed approach can be used for deriving a lumped element equivalent network for the discontinuity. The application of the discontinuity was supported by the simulated design of a miniature-sized broadband bandpass filter on MMIC with a significant fractional bandwidth (96\%) and a fairly good stop band rejection.

\section{References}

[1] C. P. Wen, "Coplanar waveguide: a surface strip transmission line suitable for nonreciprocal gyromagnetic device applications," IEEE Transactions on Microwave Theory and Techniques, vol. 17, no. 12, pp. 1087-1090, 1969.

[2] J. L. B. Walker, "Survey of European activity on coplanar waveguide," in Proceedings of the IEEE MTT-S International Microwave Symposium Digest, vol. 2, pp. 693-696, Atlanta, Ga, USA, June 1993.

[3] M. Houdart, "Coplanar lines: application to broadband microwave integrated circuits," in Proceedings of the 6th European Microwave Conference, pp. 49-53, Rome, Italy, September 1976.

[4] J. P. de Villiers and J. P. Jacobs, "Gaussian process modeling of CPW-fed slot antennas," Progress in Electromagnetics Research, vol. 98, pp. 233-249, 2009.

[5] C. Mahatthanajatuphat, P. Akkaraekthalin, S. Saleekaw, and M. Krairiksh, "A bidirectional multiband antenna with modified fractal slot fed by CPW," Progress in Electromagnetics Research, vol. 95, pp. 59-72, 2009.

[6] Z. Y. Yeh and Y. C. Chiang, "A miniature CPW balun constructed with length-reduced $3 \mathrm{~dB}$ couples and a short redundant transmission line," Progress in Electromagnetics Research, vol. 117, pp. 195-208, 2011.

[7] M. A. Habib, A. Bostani, A. Djaiz, M. Nedil, M. C. E. Yagoub, and T. A. Denidni, "Ultra wideband cpw-fed aperture antenna with wlan band rejection," Progress in Electromagnetics Research, vol. 106, pp. 17-31, 2010.

[8] C. L. Tsai, "A coplanar-strip dipole antenna for broadband circular polarization operation," Progress in Electromagnetics Research, vol. 121, pp. 141-157, 2011.

[9] W. Marynowski, P. Kowalczyk, and J. Mazur, "On the characteristic impedance definition in microstrip and coplanar lines," Progress in Electromagnetics Research, vol. 110, pp. 219$235,2010$.

[10] S. Costanzo, "Synthesis of multi-step coplanarwaveguidetomicrostrip transition," Progress in Electromagnetics Research, vol. 113, pp. 111-126, 2011.

[11] S. Gevorgian, T. Martinsson, A. Deleniv, E. Kollberg, and I. Vendik, "Simple and accurate dispersion expression for the effective dielectric constant of coplanar waveguides," IEE Proceedings of Microwaves, Antennas and Propagation, vol. 144, no. 2, pp. 145-148, 1997.

[12] R. N. Simons, Coplanar Waveguide Circuits, Components and Systems, Wiley-Interscience, New York, NY, USA, 2001.

[13] R. N. Simons and G. E. Ponchak, "Modeling of some coplanar waveguide discontinuities," IEEE Transactions on Microwave Theory and Techniques, vol. 36, no. 12, pp. 1796-1803, 1988.

[14] N. I. Dib, L. P. B. Katehi, G. E. Ponchak, and R. N. Simons, "Theoretical and experimental characterization of coplanar waveguide discontinuities for filter applications," IEEE Transactions on Microwave Theory and Techniques, vol. 39, no. 5, pp. 873-882, 1991. 
[15] M. Naghed and I. Wolff, "Equivalent capacitances of coplanar waveguide discontinuities and interdigitated capacitors using a three-dimensional finite difference method," IEEE Transactions on Microwave Theory and Techniques, vol. 38, no. 12, pp. 1808-1815, 1990.

[16] K. Beilenhoff, H. Klingbeil, W. Heinrich, and H. L. Hartnagel, "Open and short circuits in coplanar MMIC's," IEEE Transactions on Microwave Theory and Techniques, vol. 41, no. 9, pp. 1534-1537, 1993.

[17] V. Radisic, D. R. Hjelme, A. Horrigan, Z. B. Popovic, and A. R. Mickelson, "Experimentally verifiable modeling of coplanar waveguide discontinuities," IEEE Transactions on Microwave Theory and Techniques, vol. 41, no. 9, pp. 1524-1533, 1993.

[18] N. I. Dib, P. B. Katehi, G. E. Ponchak, and R. N. Simons, "Coplanar waveguide discontinuities for $\mathrm{p}-\mathrm{i}-\mathrm{n}$ diode switches and filter applications," in Proceedings of the IEEE MTT-S International Microwave Symposium Digest, vol. 1, pp. 399402, Dallas, Tex, USA, May 1990.

[19] N. Dib, J. Ababneh, and A. Omar, "CAD modeling of coplanar waveguide interdigital capacitor," International Journal of $R F$ and Microwave Computer-Aided Engineering, vol. 15, no. 6, pp. 551-559, 2005.

[20] R. Masood and S. A. Mohsin, "Optimization of the Sparameter response of a coplanar waveguide series short stub for broadband applications," in Proceedings of the $3 \mathrm{rd}$ International Conference on Communications and Electronics (ICCE'10), pp. 384-388, Nha Trang, Vietnam, August 2010.

[21] R. Masood, S. A. Mohsin, and D. Ahmed, A Novel Analysis of a Coplanar Waveguide Series Open Stub for Broadband Applications, IBCAST, Islamabad, Pakistan, 2011.

[22] D. F. Williams and S. E. Schwarz, "Design and performance of coplanar waveguide bandpass filters," IEEE Transactions on Microwave Theory and Techniques, vol. 31, no. 7, pp. 558-566, 1983.

[23] J. Hu and L. Sun, "EC-ANN models for CPW discontinuities," IEE Proceedings Microwaves, Antennas and Propagation, vol. 152, no. 5, pp. 292-297, 2005.

[24] Xi Li, Y. Shi, and Y. Ding, "Model and performance analysis of coplanar waveguide based on different oxide structure HRSi substrate," in Proceedings of the Progress in Electromagnetics Research Symposium, pp. 489-492, Xi'an, China, March 2010.

[25] G. Liu, H. Nakano, and K. Honjo, "Compact accurate scalable model for millimeter wave InP CPW with under-bridge," IEICE Electronics Express, vol. 5, no. 2, pp. 74-80, 2008.

[26] S. Gevorgian, P. L. J. Linner, and E. L. Kollberg, "CAD models for shielded multilayered CPW," IEEE Transactions on Microwave Theory and Techniques, vol. 43, no. 4, pp. 772-779, 1995.

[27] L. Zhu and W. Menzel, "Broad-band microstrip-to-CPW transition via frequency-dependent electromagnetic coupling," IEEE Transactions on Microwave Theory and Techniques, vol. 52, no. 5, pp. 1517-1522, 2004.

[28] I. Wolff, Microwave Integrated Circuits, Wiley-Interscience, New York, NY, USA, 2006.

[29] R. F. Harrington, Field Computation by Moment Methods, Macmillan, New York, NY, USA, 1968.

[30] Agilent Technologies, "Advanced Design System-Momentum," http://www.agilent.com/find/eesof-ads.

[31] CST Microwave Studio, http://www.cst.com/.

[32] K. C. Gupta, R. Garg, I. J. Bahl, and P. Bhartia, Microstrip Lines and Slotlines, Artech House, Norwood, Mass, USA, 2nd edition, 1996.

[33] R. E. Collin, Foundations for Microwave Engineering, McGrawHill, NewYork, NY, USA, 2nd edition, 1992.
[34] C. Veyres and V. F. Hanna, "Extension of the application of conformal mapping techniques to coplanar lines with finite dimensions," International Journal of Electronics, vol. 48, no. 1, pp. 47-56, 1980.

[35] H. V. Fouad, "Finite boundary corrections to coplanar stripline analysis," Electronics Letters, vol. 16, no. 15, pp. 604-606, 1980.

[36] G. Ghione and C. Naldi, "Parameters of coplanar waveguides with lower groundplane," Electronics Letters, vol. 19, no. 18, pp. 734-735, 1983.

[37] T. Kitazawa and Y. Hayashi, "Variational method for coplanar waveguide withanisotropic substrates," IEE Proceedings $H$, vol. 134, no. 1, pp. 7-10, 1987.

[38] W. Hilber, "From approximation to exact relations for characteristic impedances," IEEE Transactions on Microwave Theory and Techniques, vol. 17, no. 5, pp. 259-265, 1969. 

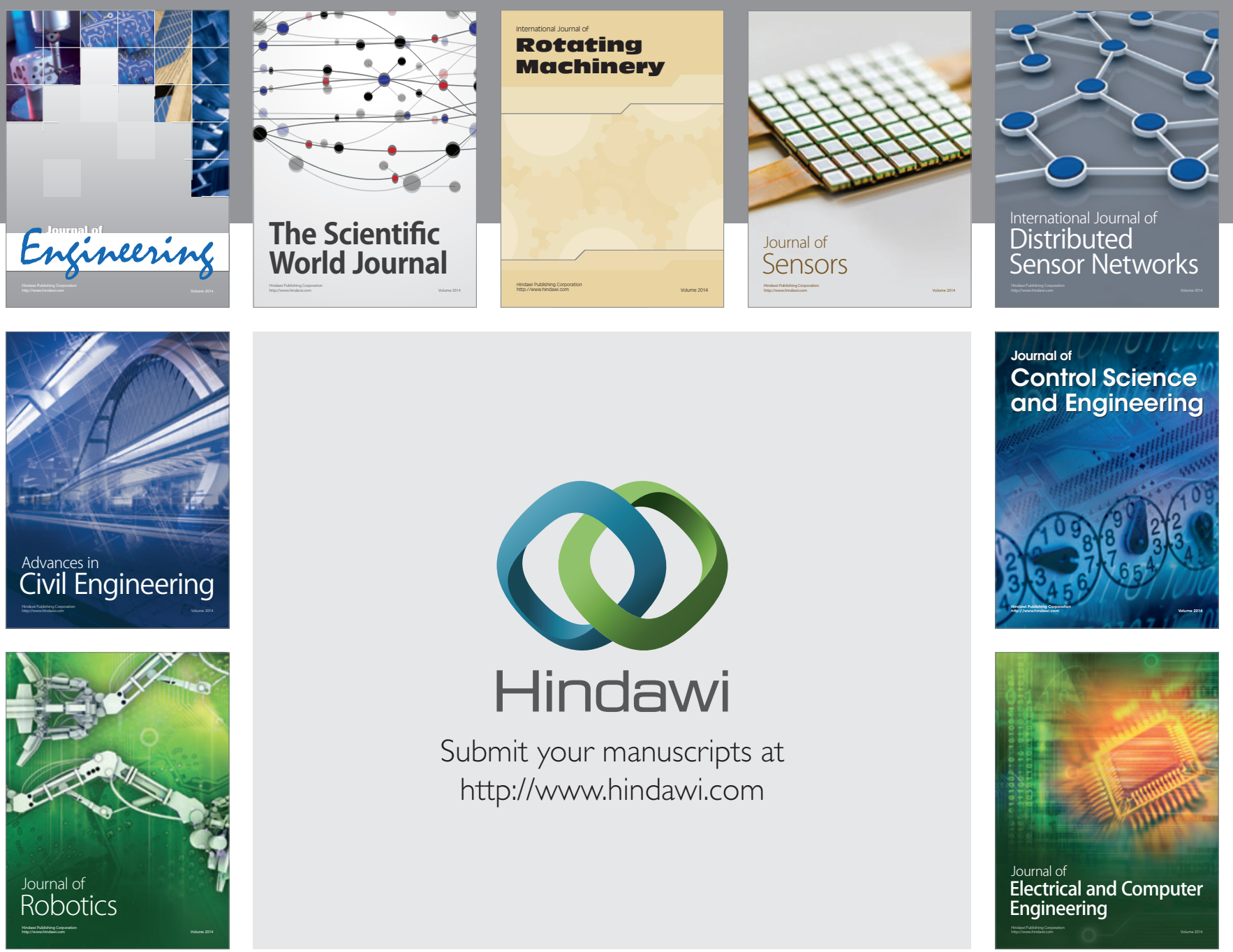

Submit your manuscripts at

http://www.hindawi.com
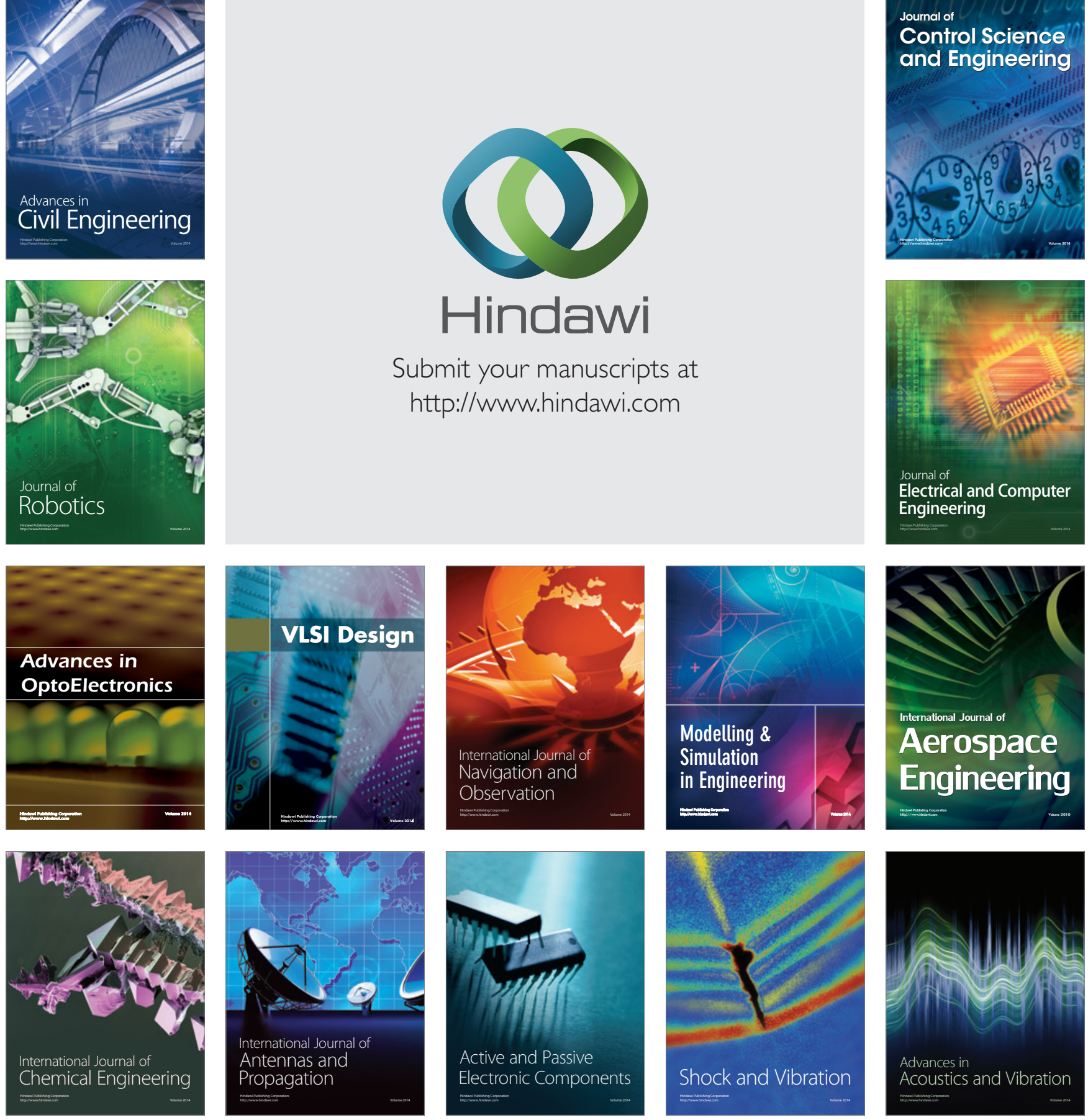